PHYSICAL REVIEW D 73, 023522 (2006)

\title{
Non-Gaussianity from broken symmetries
}

\author{
Edward W. Kolb* \\ Particle Astrophysics Center, Fermi National Accelerator Laboratory, Batavia, Illinois 60510-0500, USA \\ and Department of Astronomy and Astrophysics, Enrico Fermi Institute, University of Chicago, Chicago, Illinois 60637-1433, USA \\ Antonio Riotto ${ }^{\dagger}$ \\ CERN Theory Division CH-1211, Geneva 23, Switzerland
}

\author{
Alberto Vallinotto \\ Physics Department, The University of Chicago, Chicago, Illinois 60637-1433, USA \\ and Particle Astrophysics Center, Fermi National Accelerator Laboratory, Batavia, Illinois 60510-0500, USA
}

(Received 9 November 2005; published 26 January 2006)

\begin{abstract}
Recently we studied inflation models in which the inflaton potential is characterized by an underlying approximate global symmetry. In the first work we pointed out that in such a model curvature perturbations are generated after the end of the slow-roll phase of inflation. In this work we develop further the observational implications of the model and compute the degree of non-Gaussianity predicted in the scenario. We find that the corresponding nonlinearity parameter, $f_{N L}$, can be as large as $10^{2}$.
\end{abstract}

DOI: 10.1103/PhysRevD.73.023522

PACS numbers: $98.80 . \mathrm{Cq}$

\section{INTRODUCTION}

Inflation has become the dominant paradigm to understand the initial conditions for the density perturbations in the early Universe, which are the seeds for the Large-Scale Structure (LSS) and for Cosmic Microwave Background (CMB) temperature anisotropies [1]. In the inflationary picture, primordial density and gravity-wave fluctuations are created from quantum fluctuations and "redshifted" out of the horizon during an early period of superluminal expansion of the Universe.

Despite the simplicity of the inflationary concept, the mechanism by which cosmological curvature (adiabatic) perturbations are generated is not yet established. In the standard slow-roll inflationary scenario associated with a single inflaton field, density perturbations are due to quantum fluctuations of the inflaton itself. In the curvaton mechanism [2], the final curvature perturbation $\zeta$ is produced from an initial isocurvature mode associated with quantum fluctuations of a light scalar (other than the inflaton), the curvaton, whose energy density is negligible during inflation and which decays much after the end of inflation. Recently, we have proposed an alternative scenario for the generation of the comoving curvature perturbation [3].

The idea is that if the inflaton sector is characterized by an approximate global symmetry, the associated pseudoNambu-Goldstone boson(s) may be quantum mechanically excited during inflation. This results in different initial conditions in separate horizon volumes for the (pre)heating stage after the end of inflation. These different initial

\footnotetext{
*Electronic address: rocky@fnal.gov

${ }^{\dagger}$ Electronic address: antonio.riotto@pd.infn.it

‡Electronic address: avallino@uchicago.edu
}

conditions will give rise to fluctuations in the comoving number densities of the light relativistic states produced during the decay process and, ultimately, to CMB anisotropies. Other mechanisms for the generation of cosmological perturbations have been proposed as well, such as the inhomogeneous reheating scenario [4], ghost-inflation [5], and the D-celeration scenario [6], to mention a few.

Given all these theoretical models, it is very important to find ways to discriminate among them based on observational evidence. A precise measurement of the spectral index of comoving curvature perturbations $n_{\zeta}$ will provide a powerful constraint to slow-roll inflation models and to the standard scenario for the generation of cosmological perturbations, which predicts $\left|n_{\zeta}-1\right|$ significantly below unity. However, alternative mechanisms generally also predict a value of $n_{\zeta}$ very close to unity. Thus, even a precise measurement of the spectral index will not allow us to discriminate efficiently among them. Also, the lack of gravity-wave signals in CMB anisotropies will not provide any information about the perturbation generation mechanism, since alternative mechanisms predict an amplitude of gravity waves far too small to be detectable by future experiments aimed at observing the $B$-mode of the CMB polarization.

There is, however, a third observable which will prove fundamental in providing information about the mechanism chosen by Nature to produce the structures we see today. It is the deviation from pure Gaussian statistics, i.e., the presence of higher-order connected correlation functions of CMB anisotropies [7]. Since for every scenario there exists a well defined prediction for the strength of non-Gaussianity and its dependence on the parameters, testing the non-Gaussian level of primordial fluctuations is one of the most powerful probes of inflation [8], and is crucial to discriminate among different, but otherwise ob- 
servationally indistinguishable, mechanisms. For instance, the single-field slow-roll inflation model produces negligible non-Gaussianity, and its dominant contribution arises from the evolution of the ubiquitous second-order perturbations after inflation, which is potentially detectable with future observations of CMB temperature and polarization anisotropies. This effect must exist regardless of the specific inflationary model considered, setting a lower bound of the non-Gaussianity of cosmological perturbations. If evidence for this ubiquitous non-Gaussianity is not found experimentally, the present understanding of the evolution of cosmological perturbations will be challenged at a very deep level.

Motivated by the extreme relevance of pursuing nonGaussianity in the CMB anisotropies, in this paper we calculate the level of non-Gaussianity at large scales in the CMB anisotropies produced in the class of models where the comoving curvature perturbations are generated by broken symmetries in the inflaton sector [3]. Since we will find that in this class of models the non-Gaussianity is substantial, as a first approximation it will be possible to neglect the nonlinearity generated by gravitational dynamics in the post-inflationary phase $[9,10]$.

The paper is organized as follows. In Sec. II we present an overview of the general physical mechanism leading to curvature perturbations production during multifield inflaton decay. Section III then focuses on the determination of the comoving curvature perturbation produced when the inflaton field is characterized by a broken U(1) symmetry and when the decay process is the so-called instant preheating. In Sec. IV, we carry out the calculation of the nonlinearity parameter $f_{N L}$ for the model considered. Section $\mathrm{V}$ then generalizes such results to determine a general formula for the evaluation of the level of nonGaussianity produced whenever the potential is characterized by a broken symmetry, and summarizes the conclusions.

\section{MODEL OVERVIEW}

As a first step, let us analyze the consequences of assuming an inflationary potential characterized by a global broken symmetry for the production of curvature perturbations during the decay process of the inflaton field.

In what follows, we consider a multifield inflationary scenario where the scale factor $a(t)$ and the background inflaton fields $\phi_{i}(t)$ evolve according to the system of coupled differential equations

$$
\begin{aligned}
& \ddot{\phi}_{i}+3 H \dot{\phi}_{i}+\frac{\partial V}{\partial \phi_{i}}=0, \quad i=1, \ldots, n, \\
& H^{2}=\left(\frac{\dot{a}}{a}\right)^{2}=\frac{8 \pi}{3 M_{p}^{2}}\left[\sum_{i} \frac{\dot{\phi}_{i}^{2}}{2}+V(\vec{\phi})\right],
\end{aligned}
$$

where $\vec{\phi}=\left(\phi_{1}, \phi_{2}, \ldots, \phi_{n}\right)$.
As in the standard single-field scenario, the multifield scenario is characterized by an initial slow-roll phase during which quantum fluctuations of all the light degrees of freedom are excited, stretched to macroscopic scales, and frozen in amplitude once their wavelength exceeds the Hubble radius. It is important to note that in the singlefield scenario only fluctuations parallel to the direction of motion in field space could be generated. As was shown in Refs. [3,11], these fluctuations would later correspond to adiabatic perturbations. In the multifield case, on the other hand, fluctuations both parallel and orthogonal to the direction of motion in field space can be excited, the latter corresponding to isocurvature perturbations. In general, then, at the end of the slow-roll phase the value of the background inflaton field will have acquired a spatial dependence

$$
\vec{\phi}\left(t_{0}, x\right)=\vec{\phi}_{0}\left(t_{0}\right)+\delta \vec{\phi}\left(t_{0}, x\right)
$$

where $t_{0}$ denotes the epoch after termination of the slowroll phase but before the decay process. It is necessary to point out that up to this point in the evolution history of the Universe the only difference between the single-field and the multifield scenarios consists of the fact that field perturbations in the former case are of the adiabatic type only, while in the latter case they consist of both adiabatic and isocurvature perturbations.

Moving on to the next phase in the evolution history of the Universe, it is very important to note that the quantum fluctuations imprinted onto the value of the inflaton fields during the slow-roll phase also represent, through Eq. (2), perturbations in the initial conditions for the evolution of the background fields $\vec{\phi}$ during the decay phase. Similar to a chaotic mechanical system, if the potential $V(\vec{\phi})$ is characterized by a global broken symmetry, the system of differential equations Eqs. (1a) and (1b) then exhibits a sensitive dependence on the initial conditions. This fact, in turn, guarantees that different initial conditions will correspond to evolution histories for the background fields $\vec{\phi}(t)$ for $t \geq t_{0}$ which do not just differ from one another by a simple time translation. Then to summarize, the broken symmetry of the potential is sufficient to guarantee that the field perturbations generated during the slow-roll phase will correspond to field trajectories $\vec{\phi}(t)$ that will differ substantially in the subsequent phase of inflaton decay.

Moving on to consider the decay process, it is clear that if the inflaton fields $\vec{\phi}$ have to decay into some other particles $\chi$, then the two must necessarily be connected by an interaction Lagrangian $\mathcal{L}_{\chi \phi}$. Furthermore, this interaction Lagrangian necessarily must depend on the value of the fields involved, $\vec{\phi}$ and $\chi$. Denoting by $n_{\chi}$ the comoving number density of $\chi$ particles produced during the decay process, it is then trivial to note that such a quantity will be a (usually very complicated) functional of the evolution histories of the background fields. But since the field 
trajectories will depend on the initial conditions set at time $t_{0}$, then formally we may write

$$
n_{\chi}=F\left[\vec{\phi}\left(t_{0}\right), \ldots\right] .
$$

In a multifield scenario the perturbations generated during the slow-roll phase will appear as perturbations of the initial conditions for the evolution of the background fields during the decay stage. This will in turn result in fluctuations in the comoving number density of particles produced

$$
\delta \vec{\phi}\left(t_{0}, x\right) \Rightarrow \delta n_{\chi}\left(t_{1}, x\right)
$$

where $t_{1}>t_{0}$ denotes the end of the decay process.

To determine the curvature perturbations produced during such a decay process, it is sufficient to note that the generated energy density is proportional to the comoving number density of produced particles, $\rho_{\chi} \sim n_{\chi}$. Assuming for simplicity complete decay, an estimate of the curvature perturbation $\zeta$ generated during this phase is then

$$
\zeta \equiv-\psi-H \frac{\delta \rho_{\chi}}{\dot{\rho}_{\chi}} \approx \alpha \frac{\delta n_{\chi}}{n_{\chi}},
$$

where in the last step the spatially flat gauge has been assumed and $\alpha$ is a proportionality constant whose numerical value depends on the redshifting of the particle produced.

The level of the non-Gaussianity produced is usually specified using the nonlinearity parameter $f_{N L}$, which determines the non-Gaussian contribution to the Bardeen potential $\Phi$ according to

$$
\Phi=\Phi_{G}+f_{N L} \Phi_{G}^{2}
$$

where $\Phi_{G}$ is the Gaussian part of the Bardeen potential. It is then possible to connect $\Phi$ to $\zeta$ through $^{1}$

$$
\Phi=-\frac{3}{5} \zeta=-\frac{3 \alpha}{5} \frac{\delta n_{\chi}}{n_{\chi}}
$$

where in the last step the expression for the curvature perturbation $\zeta$ produced in the present model has been used.

It seems important to stress here that the only crucial assumption made so far concerns the global broken symmetry of the potential. If the symmetry is unbroken, it is possible to see that the perturbations of the initial conditions would produce evolution histories of the background fields $\vec{\phi}(t)$ which would only differ by a time translation: the curvature perturbations thus produced would simply represent a gauge artifact and could be gauged away by a suitable choice of the slicing and threading. The only other assumption made is that the inflaton decays into the $\chi$ field

\footnotetext{
${ }^{1}$ It is important to note that Eq. (7) connecting $\Phi$ and $\zeta$ is valid only at linear order. It is nonetheless correct to use it provided that the value of the non-Gaussianity parameter is larger than unity, which will be the case in what follows.
}

through the nonperturbative process of preheating, but this assumption is not crucial to the final conclusions that fluctuations in the initial conditions at the beginning of the decay stage would yield fluctuations in the energy density and therefore in the curvature.

Curvature perturbations produced through this mechanism can be characterized by significant degree of nonGaussianity. The next sections contain a simple example which shows how it is possible to determine the value of the non-Gaussianity parameter $f_{N L}$ in a specific case.

\section{AN ANALYTIC EXAMPLE: THE BROKEN U(1) CASE IN THE INSTANT PREHEATING SCENARIO}

Because of the general characteristics of the process being considered - a sensitive dependence of the fieldspace trajectories on the initial conditions and the nonperturbative nature of the preheating process - the general evaluation of the curvature perturbation and of the nonlinearity parameter generated usually require a numerical approach. In this section the instant preheating model of Felder et al. [12] is considered in order to obtain an analytic estimate of $f_{N L}$. It is important to stress, however, that while the choice of the specific preheating model is in part dictated by computational convenience, the results of the present section can be easily generalized to take into account different decay processes and background-field spaces.

To proceed further it is necessary to specify two details that so far have been left undetermined: the characteristics of the background-field space (dimensionality, potential, and broken symmetry) and the details of the preheating mechanism. The former determines the field trajectories $\vec{\phi}(t)$, while the latter allows the specification of the functional relating the trajectories to the comoving density of particles produced, $n_{\chi}[\vec{\phi}(t)]$.

\section{A. The Choice of the Background-Field Landscape}

Proceeding as in Ref. [3], the dimensionality of the field space is chosen to be 2 , so that the inflaton-field landscape is described by $\phi_{1}$ and $\phi_{2}$. This choice reflects the fact that curvature perturbations and possibly non-Gaussianity can arise in the simplest multifield inflationary scenario. The complex field $\phi$ can then be introduced:

$$
\phi=\phi_{1}+i \phi_{2}=|\phi| e^{i \theta} .
$$

The potential $V\left(\phi_{1}, \phi_{2}\right)$, which is characterized by a slightly broken U(1) symmetry, can be modeled close to its minimum by

$$
V\left(\phi_{1}, \phi_{2}\right)=\frac{m^{2}}{2}\left[\phi_{1}^{2}+\frac{\phi_{2}^{2}}{(1+x)}\right],
$$

where $x$ represents a measure of the symmetry breaking. This choice for the potential landscape allows one to determine analytically the field trajectories $\vec{\phi}(t)$ if the 
Hubble expansion is neglected for the short time period during which the decay process occurs (see the Appendix of Ref. [3]).

Finally, the initial conditions for the background dynamics set at the end of the slow-roll phase-and fluctuations thereof - can be equivalently expressed as $\left[\phi_{1}\left(t_{0}\right), \phi_{2}\left(t_{0}\right)\right]$ or as $\left[|\phi|\left(t_{0}\right), \theta\left(t_{0}\right)\right]$. However, since the aim of the present work is to analyze the conversion of isocurvature perturbation into adiabatic perturbations during the decay stage, and since the form of the potential is such that the motion of the field is almost completely in the radial direction, in what follows the latter choice will be adopted together with the shorthand notation $\left[\left|\phi_{0}\right|, \theta_{0}\right]$.

\section{B. The Choice of the Inflaton Decay Process}

In this analysis, the instant preheating model of Felder et al. [12] is chosen as the process through which the inflaton decays into lighter particles. Such a choice is made both because it is not unreasonable to think that the preheat field $\chi$ is coupled to other fields into which it can perturbatively decay, and because it also yields a convenient analytical expression for the functional $n_{\chi}[\vec{\phi}(t)]$.

The complex inflaton field $\phi$ is coupled to the preheat field $\chi$ through the standard preheating interaction Lagrangian $\mathcal{L}_{\chi \phi}=-\frac{1}{2} g|\phi|^{2} \chi^{2}$. The preheat field $\chi$ is also coupled to a massless fermion field $\psi$ by the interaction Lagrangian $\mathcal{L}_{\phi \psi}=h \bar{\psi} \psi \chi$, so that the perturbative decay $\chi \rightarrow \bar{\psi} \psi$ can take place. ${ }^{2}$ Applying the results of Kofman et al. [13] and of Felder et al. [12], the comoving number density of $\chi$ particles produced during the first half-oscillation of $\phi$ close to its minimum can be determined to be

$$
n_{\chi}=\frac{\left(g\left|\dot{\phi}_{*}\right|\right)^{3 / 2}}{8 \pi^{3}} \exp \left[-\frac{\pi g\left|\phi_{*}\right|^{2}}{\left|\dot{\phi}_{*}\right|}\right]
$$

where $t_{*}$ is the instant when the inflaton $\phi$ reaches the minimum of the potential $V(\phi)$ along its trajectory and $\left|\dot{\phi}_{*}\right|$ and $\left|\phi_{*}\right|$ represent the field velocity and distance from the absolute minimum of the potential at such an instant. From the interaction Lagrangian $\mathcal{L}_{\chi \phi}$ it is possible to note that as the inflaton moves away from the bottom of the potential, the preheat field is endowed with an increasing effective mass $m_{\chi}=g|\phi|$. The $\chi$ particles thus produced then acquire a large mass and decay into $\psi$ particles with a decay rate $\Gamma_{\chi \rightarrow \bar{\psi} \psi}=h^{2} m_{\chi} / 8 \pi=h^{2} g|\phi| / 8 \pi$ that is proportional to $|\phi|$, which is increasing as the background inflaton moves away from the bottom of the potential. Depending on the values of the coupling constants, the whole decay chain $\phi \rightarrow \chi \rightarrow \psi$ can proceed very efficiently, turning all the energy initially stored in the $\phi$ fields into the fermions $\psi$ in a single half-oscillation of the

\footnotetext{
${ }^{2}$ Given the large mass of the other fields involved in the decay process, the assumption of a massless $\psi$ is actually immaterial.
}

inflaton around the minimum of its potential. The fermions then rapidly thermalize and complete the reheating process.

The fluctuations in the final energy density (and thus in the CMB temperature) can then be traced back to the fluctuations in the number density of preheat particles produced during the decay process, which are in turn caused by the isocurvature perturbations generated during the slow-roll phase.

\section{THE CALCULATION OF THE NON-LINEARITY PARAMETER}

To obtain an estimate of the curvature perturbation through Eq. (5), it is necessary to find an expression for $\delta n_{\chi} / n_{\chi}$. From Eq. (10) it is straightforward to get [3]:

$$
\frac{\delta n_{\chi}}{n_{\chi}}=\left(\frac{3}{2}+\frac{\pi g\left|\phi_{*}\right|^{2}}{\left|\dot{\phi}_{*}\right|}\right) \frac{\delta\left|\dot{\phi}_{*}\right|}{\left|\dot{\phi}_{*}\right|}-\frac{2 \pi g\left|\phi_{*}\right|^{2}}{\left|\dot{\phi}_{*}\right|} \frac{\delta\left|\phi_{*}\right|}{\left|\phi_{*}\right|} .
$$

Moving on to consider the background-field trajectories, it is possible to note that the choice of the potential, Eq. (9), allows one to derive the following approximate values for $\left|\phi_{*}\right|$ and $\left|\dot{\phi}_{*}\right|$ as functions of the initial conditions $\left[\left|\phi_{0}\right|, \theta_{0}\right]$ and of the value of the symmetry breaking parameter $x$ (see the Appendix of Ref. [3]):

$$
\begin{aligned}
\left|\phi_{*}\left(\left|\phi_{0}\right|, \theta_{0} ; x\right)\right| & \approx \frac{\left|\phi_{0}\right| \pi x}{2 \sqrt{2}}\left|\sin \left(2 \theta_{0}\right)\right|, \\
\left|\dot{\phi}_{*}\left(\left|\phi_{0}\right|, \theta_{0} ; x\right)\right| & \approx m\left|\phi_{0}\right| \sqrt{1-x \sin ^{2}\left(\theta_{0}\right) .}
\end{aligned}
$$

Let us then note that the form of the potential, Eq. (9), is such that the motion of the inflaton is almost completely in the radial direction. Perturbations in the radial direction therefore correspond to adiabatic perturbations, while perturbations in the azimuthal direction - leaving the value of the potential unaltered-correspond to isocurvature perturbations. ${ }^{3}$ Since the aim of the present work is to analyze the conversion of isocurvature perturbations into adiabatic perturbations and the resulting curvature perturbations, let us then assume that during the slow-roll phase the radial part of the inflaton field induces curvature perturbations which are very suppressed. ${ }^{4}$

To obtain an estimate of the value of the non-Gaussianity parameter $f_{N L}$, the Bardeen potential $\Phi$ needs to be expressed as

$$
\Phi=f_{1}\left(\theta_{0}\right) \delta \theta_{0}+f_{2}\left(\theta_{0}\right)\left(\delta \theta_{0}\right)^{2} .
$$

Then, recalling Eq. (6), it is possible to identify

\footnotetext{
${ }^{3}$ For a more thorough analysis of this point, involving the fact that the field trajectory defines a local coordinate system in field space, we refer the reader to the discussion given in Ref. [3].

${ }^{4}$ This will occur if the mass of the (radial part of the) inflaton field is much smaller than the required value necessary to achieve a level of $10^{-5}$ in the CMB anisotropies, that is if $m \ll$ $10^{13} \mathrm{GeV}$.
} 


$$
\begin{aligned}
\Phi_{G} & =f_{1}\left(\theta_{0}\right) \delta \theta_{0}, \\
f_{N L} & =\frac{f_{2}\left(\theta_{0}\right)\left(\delta \theta_{0}\right)^{2}}{\Phi_{G}^{2}}=\frac{f_{2}\left(\theta_{0}\right)}{f_{1}^{2}\left(\theta_{0}\right)} .
\end{aligned}
$$

It is also necessary to point out that while in Ref. [3] we performed the calculation also including terms of order $x^{2}$ and $x^{3}$, in what follows only terms linear in the symmetry breaking parameter will be considered. Let us then notice that the term

$$
\frac{\pi g\left|\phi_{*}\right|^{2}}{\left|\dot{\phi}_{*}\right|}=\frac{\pi^{3} g x^{2}\left|\phi_{0}\right| \sin ^{2}\left(2 \theta_{0}\right)}{8 m \sqrt{1-x \sin ^{2}\left(\theta_{0}\right)}},
$$

is of order $x^{2}$, which then means that every term that contains it as a multiplying factor can safely be neglected at linear order in $x$. This then necessarily implies that

$$
\Phi \approx-\frac{9 \alpha}{10} \frac{\delta\left|\dot{\phi}_{*}\right|}{\left|\dot{\phi}_{*}\right|} .
$$

From Eqs. (12a) and (12b) it is then possible to derive the second-order expressions for

$$
\begin{aligned}
\frac{\delta\left|\phi_{*}\right|}{\left|\phi_{*}\right|}= & 2 \frac{\cos \left(2 \theta_{0}\right)}{\sin \left(2 \theta_{0}\right)} \delta \theta_{0}-\delta \theta_{0}^{2}, \\
\frac{\delta\left|\dot{\phi}_{*}\right|}{\left|\dot{\phi}_{*}\right|}= & -\frac{x}{2\left[1-x \sin ^{2}\left(\theta_{0}\right)\right]}\left\{\sin \left(2 \theta_{0}\right) \delta \theta\right. \\
& \left.+\left[\cos \left(2 \theta_{0}\right)+\frac{x \sin ^{2}\left(2 \theta_{0}\right)}{4\left[1-x \sin ^{2}\left(\theta_{0}\right)\right]}\right] \delta \theta_{0}^{2}\right\},
\end{aligned}
$$

where the expression for $\delta\left|\phi_{*}\right| /\left|\phi_{*}\right|$ has been added for completeness. We then have

$$
\begin{aligned}
\Phi= & \frac{9 \alpha x}{20\left[1-x \sin ^{2}\left(\theta_{0}\right)\right]}\left\{\sin \left(2 \theta_{0}\right) \delta \theta\right. \\
& \left.+\left[\cos \left(2 \theta_{0}\right)+\frac{x \sin ^{2}\left(2 \theta_{0}\right)}{4\left[1-x \sin ^{2}\left(\theta_{0}\right)\right]}\right] \delta \theta_{0}^{2}\right\},
\end{aligned}
$$

which then leads to the identification

$$
\begin{aligned}
f_{1}\left(\theta_{0}\right)= & \frac{9 \alpha x}{20\left[1-x \sin ^{2}\left(\theta_{0}\right)\right]} \sin \left(2 \theta_{0}\right), \\
f_{2}\left(\theta_{0}\right)= & \frac{9 \alpha x}{20\left[1-x \sin ^{2}\left(\theta_{0}\right)\right]}\left[\cos \left(2 \theta_{0}\right)\right. \\
& \left.+\frac{x \sin ^{2}\left(2 \theta_{0}\right)}{4\left[1-x \sin ^{2}\left(\theta_{0}\right)\right]}\right] .
\end{aligned}
$$

It is then immediate to find that

$$
\begin{aligned}
f_{N L}= & \frac{20}{9 \alpha} \frac{\left[1-x \sin ^{2}\left(\theta_{0}\right)\right]}{x \sin ^{2}\left(2 \theta_{0}\right)}\left[\cos \left(2 \theta_{0}\right)\right. \\
& \left.+\frac{x}{4} \frac{\sin ^{2}\left(2 \theta_{0}\right)}{\left[1-x \sin ^{2}\left(\theta_{0}\right)\right]}\right] \\
\approx & \frac{20}{9 \alpha} \frac{\cos \left(2 \theta_{0}\right)}{x \sin ^{2}\left(2 \theta_{0}\right)}
\end{aligned}
$$

where in the last expression only the leading term has been considered. ${ }^{5}$ Figure 1 shows the values of $f_{N L}$ obtained for $x=10^{-1}$ and for $x=10^{-2}$. It is then very interesting to note that this particular model is characterized by a value of the nonlinearity parameter which is inversely proportional to the value of the symmetry breaking: in general slightly broken symmetries should then produce mild curvature perturbations characterized by large nonGaussianity.

\section{GENERAL RESULT FOR $f_{N L}$ AND CONCLUSIONS}

The results of the previous section were obtained under very specific assumptions: a broken $U(1)$ symmetry and the instant preheating mechanism. It is nonetheless possible to generalize the previous approach to obtain a general formula to estimate the value of the nonlinearity parameter of the curvature perturbations produced in a multifield scenario by a generic decay process.

As it was shown in Ref. [3], the initial conditions perturbation $\delta \vec{\phi}\left(t_{0}, x\right)$ can in fact be decomposed into an adiabatic component $\delta \phi_{\|}$parallel to the field velocity $\hat{u}_{\|} \equiv d \vec{\phi} / d t$ and into an isocurvature component $\delta \phi_{\perp}$ perpendicular to it and directed along the unit vector $\hat{u}_{\perp}$. As in Ref. [3], it is assumed here as well that the adiabatic perturbations generated during the slow-roll phase of inflation are actually negligible compared to the isocurvature component

$$
\delta \phi_{\perp} \gg \delta \phi_{\|}
$$

This immediately leads to the following estimate for the Gaussian curvature perturbation

$$
\Phi_{G} \approx-\frac{3 \alpha}{5 n_{\chi}} \frac{\partial n_{\chi}}{\partial \phi_{\perp}} \delta \phi_{\perp}
$$

It is important to stress that the functional relating the comoving number density of particles produced during the decay process to the background trajectory is in general very complicated, usually requiring numerical solutions to formulate quantitative predictions. Furthermore, if the potential is characterized by a broken symmetry then the background-field trajectories may also be characterized by a sensitive dependence on the initial conditions and therefore may exhibit chaotic behavior. All these factors then conspire to make the evaluation of $\Phi$ rather involved.

Recalling Eqs. (6) and (7) and expanding $\delta n_{\chi} / n_{\chi}$ to second order and neglecting the terms containing powers of $\delta \phi_{\|}$, the Bardeen potential turns out to be

\footnotetext{
${ }^{5}$ It is in fact important to note that if we wanted to perform a calculation exact up to $x^{0}$ order we should also consider a term arising from the $\delta\left|\phi_{*}\right| /\left|\phi_{*}\right|$ factor.
} 


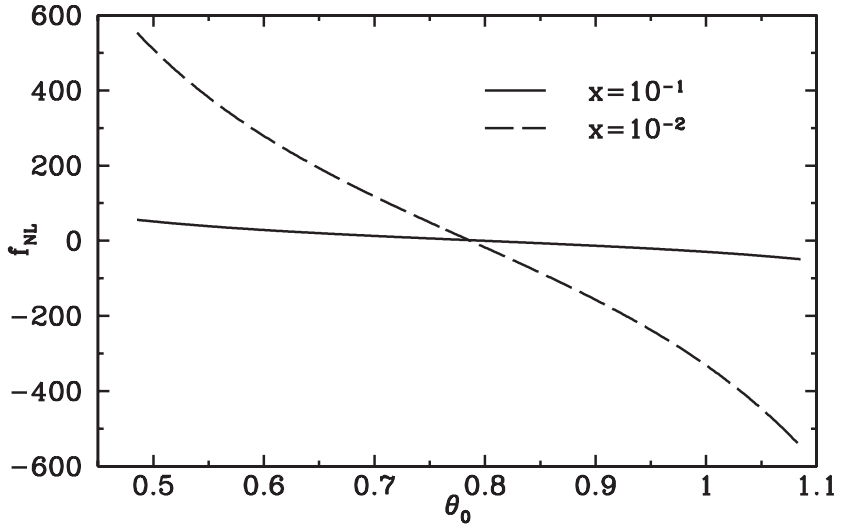

FIG. 1. Value of $f_{N L}$ for values of the symmetry breaking parameter of $x=10^{-1}$ (solid curve) and $x=10^{-2}$ (dashed curve). It seems important to stress that only a very small interval in the initial conditions $\theta_{0}$ will yield insignificant nonGaussianity.

$$
\Phi=-\frac{3 \alpha}{5 n_{\chi}}\left[\frac{\partial n_{\chi}}{\partial \phi_{\perp}} \delta \phi_{\perp}+\frac{1}{2} \frac{\partial^{2} n_{\chi}}{\partial \phi_{\perp}^{2}}\left(\delta \phi_{\perp}\right)^{2}\right]
$$

which then allows one to obtain the following general expression for the nonlinearity parameter

$$
f_{N L}=-\frac{5 n_{\chi}}{6 \alpha} \frac{\partial^{2} n_{\chi} / \partial \phi_{\perp}^{2}}{\left(\partial n_{\chi} / \partial \phi_{\perp}\right)^{2}} .
$$

It is then clear that depending on the relative magnitudes of the derivatives of $n_{\chi}$, significant or negligible nonlinearity can be produced by a multifield potential characterized by a global broken symmetry. In particular, it is straightforward to obtain the following condition

$$
f_{N L} \geq 1 \Leftrightarrow \frac{1}{n_{\chi}} \frac{\partial^{2} n_{\chi}}{\partial \phi_{\perp}^{2}} \geq \frac{2 \alpha}{n_{\chi}^{2}}\left(\frac{\partial n_{\chi}}{\partial \phi_{\perp}}\right)^{2} .
$$

which, given the chaotic nature of the background-field trajectories and the exponential production of particles in the resonances characteristics of the preheating processes, can possibly be satisfied by a large class of models.

A few comments are finally in order. The level of nonGaussianity predicted by the model at hand can be rather sizable, values of $f_{N L} \sim 100$ can be easily achieved. As we have already mentioned in the Introduction, in the computation of the nonlinearity parameter $f_{N L}$ we have neglected the nonlinear gravitational contribution coming from the post-inflationary evolution. Since they amount to a contribution to $f_{N L}$ of order unity [8], it follows that the estimate of $f_{N L}$ will be valid only in the regime $f_{N L} \gg 1$. In other words, the validity of the estimate requires that the global symmetry is slightly broken, that is $x \ll 1$. The fact that the level of non-Gaussianity is inversely proportional to the parameter $x$, which gives a measure of the breaking of the global symmetry within the inflaton sector, is reminiscent of what happens for the curvaton scenario [14]. There the non-Gaussianity is inversely proportional to the parameter $r$ which measures the energy density contribution of the curvaton to the total one at the time of curvaton decay. Since present WMAP data impose a bound $\left|f_{N L}\right| \lesssim 100$, this translates into a weak bound on the breaking parameter

$$
x \gtrsim 10^{-2} \tan \left(2 \theta_{0}\right) \sin \left(2 \theta_{0}\right) .
$$

Since future tests of the angular bispectrum of temperature anisotropy alone could detect non-Gaussianity as small as $\left|f_{N L}\right| \sim 5$ [8], it would be interesting to generalize the computation performed in this paper to include the contribution of gravitational nonlinearities which render the nonlinearity parameter $f_{N L}$ momentum-dependent. As explicitly shown in Ref. [10], this dependence augments the non-Gaussian signal at large multipoles and may be therefore crucial in testing large symmetry breaking patterns in the inflaton sector.

\section{ACKNOWLEDGMENTS}

A. R. is on leave of absence from INFN, Padova (Italy). E. W. K. and A. V. were supported in part by NASA grant NAG5-10842 and by the Department of Energy.
[1] D. H. Lyth and A. Riotto, Phys. Rep. 314, 1 (1999).

[2] K. Enqvist and M. S. Sloth, Nucl. Phys. B626, 395 (2002); D. Lyth and D. Wands, Phys. Lett. B 524, 5 (2002); T. Moroi and T. Takahashi, Phys. Lett. B 522, 215 (2001); 539, 303 (2002).

[3] E. W. Kolb, A. Riotto, and A. Vallinotto, Phys. Rev. D 71, 043513 (2005).

[4] G. Dvali, A. Gruzinov, and M. Zaldarriaga, Phys. Rev. D 69, 023505 (2004).

[5] N. Arkani-Hamed, H.C. Cheng, M. A. Luty, and S. Mukohyama, J. High Energy Phys. 05 (2004) 074.
[6] E. Silverstein and D. Tong, Phys. Rev. D 70, 103505 (2004).

[7] E. Komatsu et al., Astrophys. J. Suppl. 148, 119 (2003).

[8] N. Bartolo, E. Komatsu, S. Matarrese, and A. Riotto, Phys. Rep. 402, 103 (2004).

[9] N. Bartolo, S. Matarrese, and A. Riotto, J. High Energy Phys. 04 (2004) 006; N. Bartolo, S. Matarrese, and A. Riotto, J. Cosmol. Astropart. Phys. 01 (2004) 003; N. Bartolo, S. Matarrese, and A. Riotto, Phys. Rev. Lett. 93, 231301 (2004); N. Bartolo, S. Matarrese, and A. Riotto, J. Cosmol. Astropart. Phys. 08 (2005) 010; 
[10] M. Liguori, F. K. Hansen, E. Komatsu, S. Matarrese, and A. Riotto, astro-ph/0509098.

[11] C. Gordon, D. Wands, B. A. Bassett, and R. Maartens, Phys. Rev. D 63, 023506 (2001).

[12] G. N. Felder, L. Kofman, and A. D. Linde, Phys. Rev. D
59, 123523 (1999).

[13] L. Kofman, A. D. Linde, and A. A. Starobinsky, Phys. Rev. D 56, 3258 (1997).

[14] D. H. Lyth, C. Ungarelli, and D. Wands, Phys. Rev. D 67, 023503 (2003). 